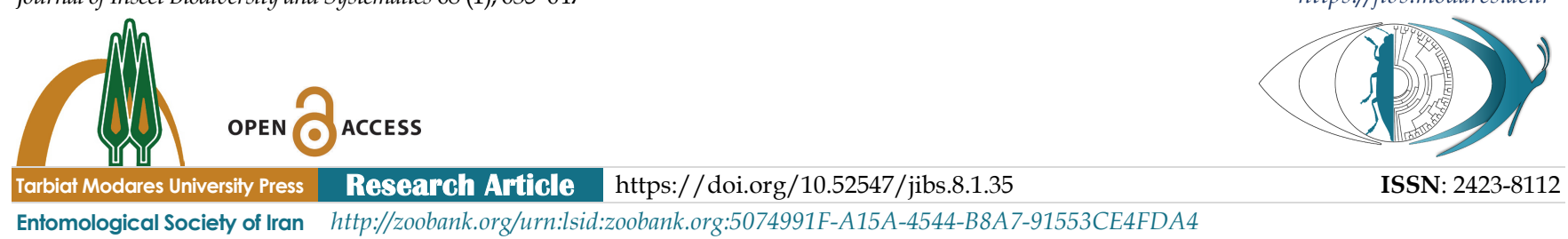

\title{
Associated Bracon Fabricius (Hymenoptera: Braconidae) with flower-heads of Asteraceae in Iran
}

\author{
Alireza Pourhaji \\ Plant Protection Research Department, East-Azarbaijan Agricultural and Natural Resources Research Center, Agricultural \\ Research, AREEO, Tabriz, Iran. \\ هa_pourhaji@yahoo.com; (iD https://orcid.org/0000-0002-6847-3213
}

Hossein Lotfalizadeh

Plant Protection Research Department, East-Azarbaijan Agricultural and Natural Resources Research Center, Agricultural Research, AREEO, Tabriz, Iran.

هhlotfalizadeh@gmail.com; (iD https://orcid.org/0000-0002-7927-819X

Naser Kasebi

Department of Forest and Rangeland, East-Azarbaijan Agricultural and Natural Resources Research \& Education Center, Agricultural Research, AREEO, Tabriz, Iran.

$\triangle$ \nkassebi46@gamil.com; (iD https://orcid.org/0000-0001-7809-333X

\section{Babak Gharali}

Plant Protection Research Department, Qazvin Agricultural and Natural Resources Research Center, Agricultural Research, AREEO, Qazvin, Iran

$\triangle$ bgharaei@yahoo.com; (1D https://orcid.org/0000-0003-2490-156X

Ali Ameri

Department of Insect Taxonomy, Iranian Research Institute of Plant Protection, Tehran, Iran. هaliameri_5@yahoo.com; (iD https://orcid.org/0000-0003-2372-8494

Received:

16 June, 2021

Accepted:

18 November, 2021

Published:

05 January, 2022

Subject Editor:

Kees van Achterberg
ABSTRACT. A study was conducted to determine the Braconinae (Hym., Braconidae) parasitoid wasps, associated with Asteraceae in the northwest of Iran during 2013-2015. Ten species were reared from flower-heads of Asteraceae: Bracon bipartitus Wesmael, 1838; B. leptus Marshall, 1897; B. luteator Spinola, 1808; B. mariae Dalla Torre, 1898; B. pectoralis Wesmael, 1838; B. subrugosus Szépligeti, 1901; B. talyshicus Tobias, 1976; B. trucidator Marshall, 1888; B. tschitscherini Kokujev, 1904 and B. urinator (Fabricius, 1798), of which one species (i.e., B. talyshicus) is recorded for the first time from Iran. We also reared some non-frugivorous Tephritidae feeding on the collected flower-heads that can be possible host of these braconids. General distribution and biological associations for all species that have been collected in this study are compiled.

Key words: Host, distribution, parasitoid, Braconinae, Iran

Citation: Pourhaji, A., Lotfalizadeh, H., Kasebi, N., Gharali, B. \& Ameri, A. (2022) Associated Bracon Fabricius (Hymenoptera: Braconidae) with flower-heads of Asteraceae in Iran. Journal of Insect Biodiversity and Systematics, 8 (1), $035-047$.

\section{INTRODUCTION}

Hymenoptera is one of the most diverse insect orders worldwide, with more than 156,365 known species (Aguiar et al., 2013; Yu et al., 2016; Sharkey et al., 2021). The family Braconidae (Hymenoptera: Ichneumonoidea) is the second largest family of Hymenoptera, with more than 21,626 valid species

Corresponding author: Pourhaji, A., E-mail: a_pourhaji@yahoo.com

Copyright (C) 2022, Pourhaji et al. This is an open access article distributed under the terms of the Creative Commons NonCommercial Attribution License (CC BY NC 4.0), which permits Share - copy and redistribute the material in any medium or format, and Adapt - remix, transform, and build upon the material, under the Attribution-NonCommercial terms. 
belonging to more than 1,106 genera (Yu et al., 2016; Sharkey et al., 2021), described worldwide, that probably is less than $20 \%$ of the total diversity worldwide (van Achterberg, 2014). Up to now 26 subfamilies 141 genera and about 900 species were recorded from Iran (Farahani et al., 2016; Abdoli et al., 2019a; Ameri et al., 2020; Zargar et al., 2020; Abdoli et al., 2021a, 2021b; Dolati et al., 2021). Biologically, the vast majority of braconids are primary parasitoids of other insects, especially upon the larval stages of Coleoptera, Diptera, and Lepidoptera (Shaw \& Huddleston, 1991; van Achterberg, 1993; Wharton, 1993), some hemimetabolous insects like aphids (Starý, 1970), web spinners (Shaw \& Edgerly, 1986) and plant-bugs (Wharton, 1993; Varis \& van Achterberg, 2001). The Braconinae of Iran was summarized in the recent checklist (Farahani et al., 2016), which lists 11 genera and 115 species. Rahmani et al. (2017) reported eight new records of this subfamily and increased number of Bracon species to 94 in Iran.

The fruit flies of the subfamily Tephritinae (Dipt.: Tephritidae), usually attack the flower heads of wide range of plant taxa including Aquifoliaceae, Scrophulariaceae, Verbenaceae, and mainly of Asteraceae as leaf miners or gall formers (Norrbom, 2010; Uchôa \& Niccio, 2010). So far, 132 species of this subfamily is recorded from Iran (Mohammadzade-Namin \& Korneyev, 2018). Čapek \& Zwolfer (1990) reported 31 species of the braconids within eight subfamilies: Braconinae, Rogadinae, Cheloninae, Microgastrinae, Agathidinae, Macrocentrinae, Helconinae and Alysiinae in association with Asteraceae. The associated parasitoid species of non-frugivorous Tephritidae have been already known in Braconidae, Chalcididae, Diapriidae, Eulophidae, Eurytomidae, Figitidae, and Pteromalidae (Wharton et al., 1998; Sivinski et al., 2000; Ovruski et al., 2004; Gates et al., 2008; Pourhaji et al., 2016, 2020). About 200 species of Braconidae have been obtained from fugivorous and non-frugivorous tephritids throughout the world (Wharton \& Yoder, 2014). Among them, there are many species that parasitize non-frugivorous tephritid flies on Asteracease (Edwards et al., 1996; Basheer et al., 2014). Lotfalizadeh and Gharali (2014) listed 12 parasitoids species on Carthamus tinctorius L. that infested by non-frugivorous Tephritidae in Iran, of which, three species have belonged to family Braconidae. The objective of this study is to improve our knowledge about the Braconid associated with Asteraceae that may parasitize non-frugivorous tephritid flies (Diptera: Tephritidae).

\section{MATERIAL AND METHODS}

This study was carried out in 14 localities (Fig. 1) of East and West-Azarbaijan provinces in the

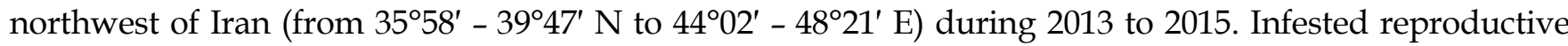
organs of 21 species of Asteraceae were widely collected and transferred to the entomology laboratory of Plant Protection Research Department, East-Azarbaijan Agricultural and Natural Resources Research Center. Before incubation, occurrence of other insects on reproductive organs was inspected. Rearing was done in the cylindrical plastic boxes $(9 \mathrm{~cm}$ diameter and $13 \mathrm{~cm}$ Height $)$ at $25 \pm 2{ }^{\circ} \mathrm{C}$ until the adults of flies and/or their parasitoids were emerged. Tephritids were identified using Korneyev \& White (1999) and their associated braconids were identified using keys and descriptions provided by Tobias (1986), Beyarslan \& Fischer (1990) and Papp (1966, 1968, 1969a, 1969b). Identifications were confirmed or corrected by Dr. K. Samartsev (Zoological Institute of the Russian Academy of Sciences, Saint Petersburg). The general distribution of the recorded Bracon species followed $\mathrm{Yu}$ et al. (2016). External morphology was illustrated using an Olympus ${ }^{\circledR} \mathrm{SZH}$, equipped with a Canon ${ }^{\circledR}$ A720 digital camera. All specimens are deposited in the insect collection of the Department of Plant Protection, East-Azarbaijan Agricultural and Natural Resources Research and Education Center, Tabriz, and one speciemen of each species in the Zoological Institute of the Russian Academy of Sciences, Saint Petersburg, Russia (ZISP). 


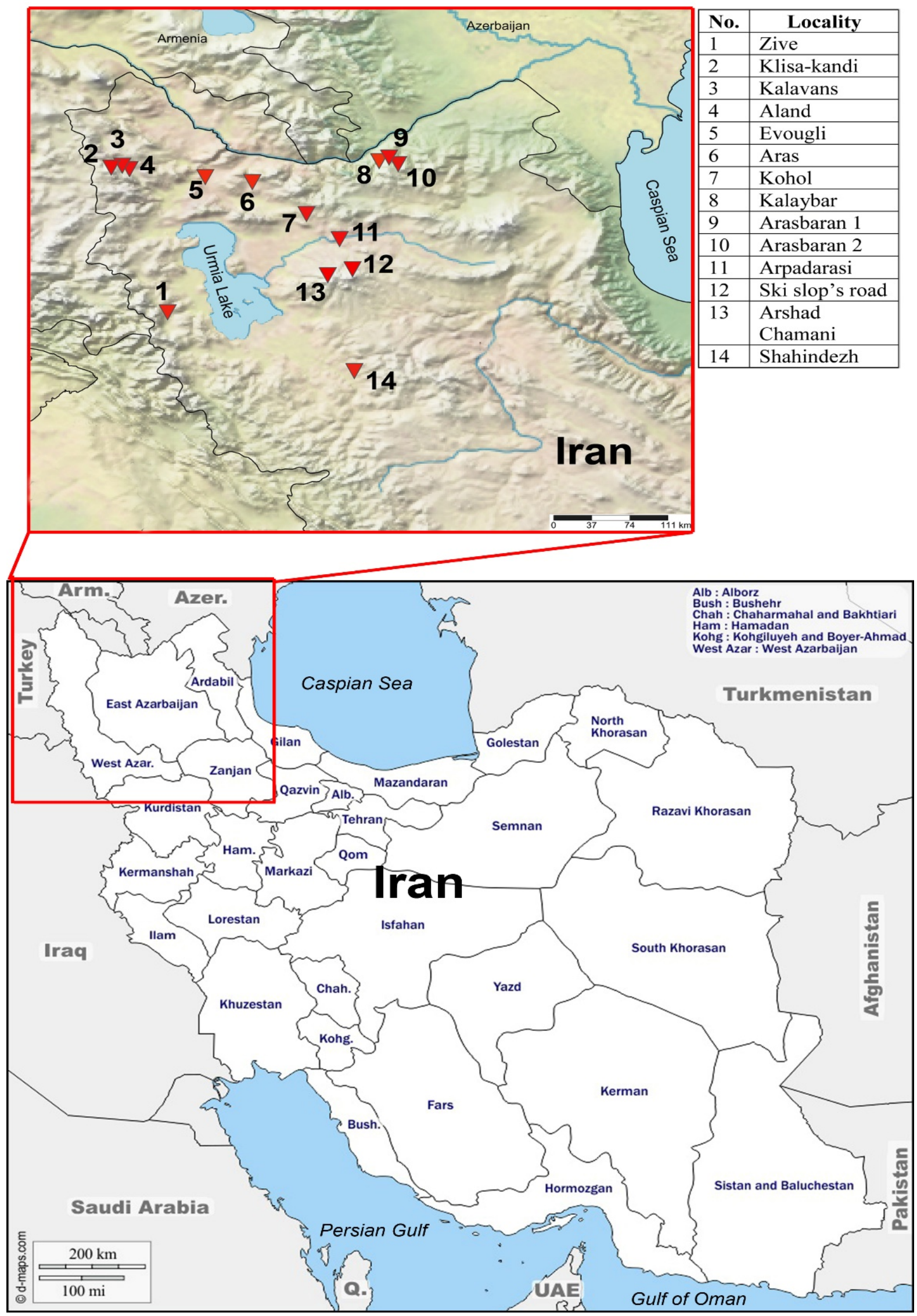

Figure 1. Collection localities of associated Braconidae with flower-heads of Asteraceae in the northwest of Iran. 


\section{RESULTS}

A total of 90 specimens of braconid parasitoids were reared from 21 species of Asteraceae that were infested by 15 species of non-frugivorous tephritids (Table 1). Identified Bracon species (Hymenoptera: Braconidae: Braconinae) are listed below.

\section{Bracon bipartitus Wesmael, 1838}

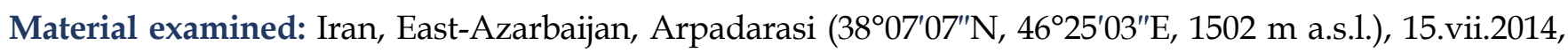
10, ex Lactuca serriola, infested with Hypenidium robrowskii (Becker, 1908); West-Azarbaijan, Sardasht $\left(36^{\circ} 28^{\prime} 47^{\prime \prime} \mathrm{N}, 45^{\circ} 19^{\prime} 10^{\prime \prime} \mathrm{E}, 1323 \mathrm{~m}\right.$ a.s.l.), 9.viii.2014, 1今', ex Echinops sp. infested with Tephritomiya lauta (Loew, 1969), leg.: A.R. Pourhaji.

Distribution in Iran: Kerman, Kermanshah, North Khorasan, Sistan-o Baluchestan (Rahmani et al., 2017) and Khuzestan (Zargar et al., 2020).

General distribution: Palaearctic.

\section{Bracon leptus Marshall, 1897}

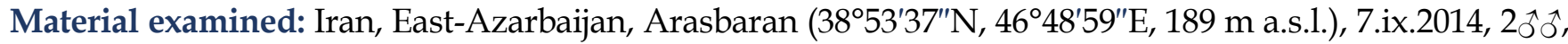
1우, ex Causinia sp., leg.: A.R. Pourhaji.

Distribution in Iran: Hormozgan (Ameri et al., 2014).

General distribution: Palaearctic.

\section{Bracon luteator Spinola, 1808}

Material examined: Iran, East-Azarbaijan, Arasbaran $\left(38^{\circ} 51^{\prime} 42^{\prime \prime} \mathrm{N}, 46^{\circ} 52^{\prime} 45^{\prime \prime} \mathrm{E}, 1696 \mathrm{~m}\right.$ a.s.1.), 7.ix.2014,1웅 ex Cirsium spectabile; Ivand $\left(38^{\circ} 21^{\prime} 20^{\prime \prime} \mathrm{N}, 4^{\circ} 07^{\prime} 21^{\prime \prime} \mathrm{E}, 1692 \mathrm{~m}\right.$ a.s.1.), 1.viii.2014, 1의 ex

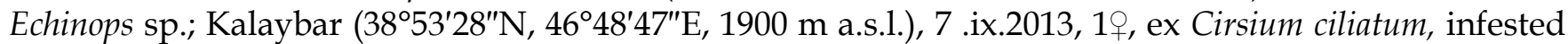
with Xyphosia mililaria (Schrank, 1781); Arshad chamani $\left(37^{\circ} 45^{\prime} 04^{\prime \prime} \mathrm{N}, 46^{\circ} 18^{\prime} 51^{\prime \prime} \mathrm{E}, 2847 \mathrm{~m}\right.$ a.s.1.), 27.vii.2014, 10ิ, ex Onopordum sp., infested with Tephritis postica (Loew, 1844); Kohol (38²6'01"N, $46^{\circ} 10^{\prime} 22^{\prime \prime} \mathrm{E}, 2282 \mathrm{~m}$ a.s.1.), 5.viii.2014, 1웅 ex Onopordum acanthium, infested with T. postica; Kalaybar (38 53'28" N, 46²4'47" E, 1900 m a.s.1.), 7.ix.2013, 1ㅇ, ex Cirsium vulgare, infested with Terellia serratula

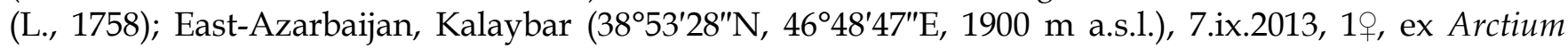
lappa, infested with Tephritomiya lauta (Loew, 1969); West-Azarbaijan, Evougli $\left(38^{\circ} 44^{\prime} 01^{\prime \prime} \mathrm{N}, 45^{\circ} 14^{\prime} 41^{\prime \prime} \mathrm{E}\right.$, 971 m a.s.1.), 4.vii.2014,10, ex Centaurea iberica, infested with Urophora sp.; leg.: A.R. Pourhaji.

Distribution in Iran: Reported from East-Azarbaijan, Chaharmahal-e Bakhtiari, Ilam (Lotfalizadeh \& Gharali, 2014) and Khuzestan (Zargar et al., 2020).

General distribution: Palaearctic.

\section{Bracon mariae Dalla Torre, 1898}

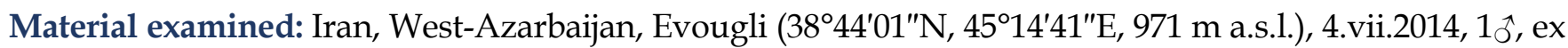
Centaurea iberica, infested with Chaetorellia carthami Stackelberg, 1929 and Chaetorellia conjuncta (Becker, 1913); Onopordum sp., infested with Tephritis postica (Loew, 1844) and Urophora sp., leg.: A.R. Pourhaji.

Distribution in Iran: no specific locality cited (Tobias, 1986)

General distribution: Palaearctic.

Bracon pectoralis Wesmael, 1838

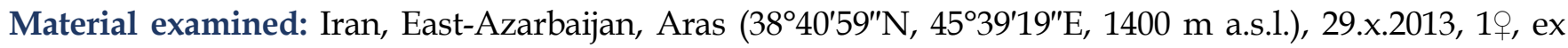
Carlina vulgare, leg.: A.R. Pourhaji. 
Distribution in Iran: Hormozgan (Ameri et al., 2015), Guilan, Qazvin (Zargar et al., 2015) and Khuzestan (Zargar et al., 2020).

General distribution: Palaearctic.

Bracon subrugosus Szépligeti, 1901

Material examined: Iran, West-Azarbaijan, Kilisa-kandi $\left(38^{\circ} 50^{\prime} 39^{\prime \prime} \mathrm{N}, 4^{\circ} 25^{\prime} 24^{\prime \prime} \mathrm{E}, 2319 \mathrm{~m}\right.$ a.s.1.), 7.vii.2015, 10ิ, ex Cirsium echinus, infested with Tephritis cometa (Loew, 1840), leg.: A.R. Pourhaji.

Distribution in Iran: Khuzestan (Zargar et al., 2020).

General distribution: Palaearctic.

Bracon (Lucobracon) talyshicus Tobias, 1974 (Figs 2-3)

Material examined: Iran, West-Azarbaijan, Kalavans (38²4'25'N $4^{\circ} 39^{\prime 2} 26^{\prime \prime} \mathrm{E}, 2044 \mathrm{~m}$ a.s.l.), 17.vii.2014,

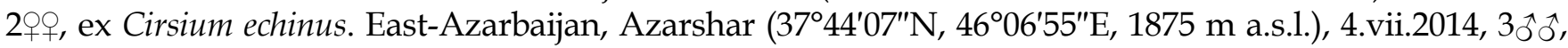
1이 ex Echinops sp., infested with Tephritomiya lauta (Loew, 1969), leg: A.R. Pourhaji.

Note. This specimens were reared on Echinops sp. that was infested by Tephritomyia lauta (Loew, 1869) (Dipt.: Tephritidae) and Cirsium echinus for the first time. There was not any host record for this parasitoid (Yu et al., 2016).

Distribution in Iran: East Azarbaijan and West Azarbaijan

General distribution: Azerbaijan, Iran (New record).

\section{Bracon trucidator Marshall, 1888}

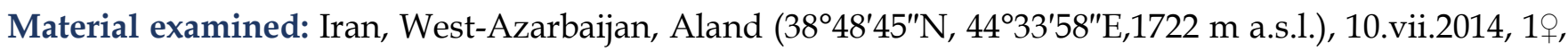
ex Onopordum sp., infested with Urophora terebrans (Loew, 1850), East-Azarbaijan, Arasbaran (38 51'42"N, 4652'45"E, $1696 \mathrm{~m}$ a.s.1.), 29.viii.2014, 10, 1ㅇ, ex Cirsium arvensis, infested with Chaetorellia sp., Ski slope's road $\left(37^{\circ} 49^{\prime} 12^{\prime \prime} \mathrm{N}, 46^{\circ} 31^{\prime} 48^{\prime \prime} \mathrm{E}, 2440 \mathrm{~m}\right.$ a.s.1.), 1.ix.2014, 1今, ex Cirsium sp., infested with Terellia nigripalpis Hendel, 1927, Arshad chamani $\left(37^{\circ} 45^{\prime} 40^{\prime \prime} \mathrm{N}, 46^{\circ} 18^{\prime} 51^{\prime \prime} \mathrm{E}, 2847 \mathrm{~m}\right.$ a.s.1.), 27.vi.2014, 10ิ, ex Onopordum acanthium, infested with Urophora sp., Kohol $\left(38^{\circ} 22^{\prime} 3^{\prime \prime} \mathrm{N}, 46^{\circ} 07^{\prime} 42^{\prime \prime} \mathrm{E}, 1729 \mathrm{~m}\right.$ a.s.l.), 1.viii.2014, 1으, ex Arctium lappa, infested with Tephritis bardanae (Schrank, 1830), leg.: A.R. Pourhaji.

Distribution in Iran: Hormozgan (Ameri et al., 2015), Kerman, Kermanshah and North Khorasan (Rahmani et al., 2017).

General distribution: Palaearctic.

\section{Bracon tschitscherini Kokujev, 1904}

Material examined: Iran, West-Azarbaijan, Shahindezh $\left(36^{\circ} 48^{\prime} 39^{\prime \prime} \mathrm{N}, 46^{\circ} 32^{\prime} 46^{\prime \prime} \mathrm{E}, 1614 \mathrm{~m}\right.$ a.s.l.), 17.vi.2014, 2o+o, ex Echinops sp., infested with Tephritomiya lauta (Loew, 1969), leg: A.R. Pourhaji.

Distribution in Iran: West Azarbaijan province (Ghahari \& Fischer, 2011).

General distribution: Palaearctic.

Bracon urinator (Fabricius, 1798)

Material examined: Iran, West-Azarbaijan, Kilisa-Kandi $\left(38^{\circ} 50^{\prime} 39^{\prime \prime} \mathrm{N}, 44^{\circ} 2^{\prime} 24^{\prime \prime} \mathrm{E}, 2319\right.$ m a.s.l.), 7.viii.2015, 10, ex Cirsium congestum, infested with Tephritomiya lauta (Loew, 1969); Arasbaran (3851'42"N, 4652'45"E, 1696 m a.s.1.), 29.viii.2014, 1``, ex Cirsium spectabilis, leg: A.R. Pourhaji.

Distribution in Iran: No specific locality cited (Beyarslan et al., 2005) and Khuzestan (Zargar et al., 2020).

General distribution: Palaearctic, Afrotropical and Oriental regions. 

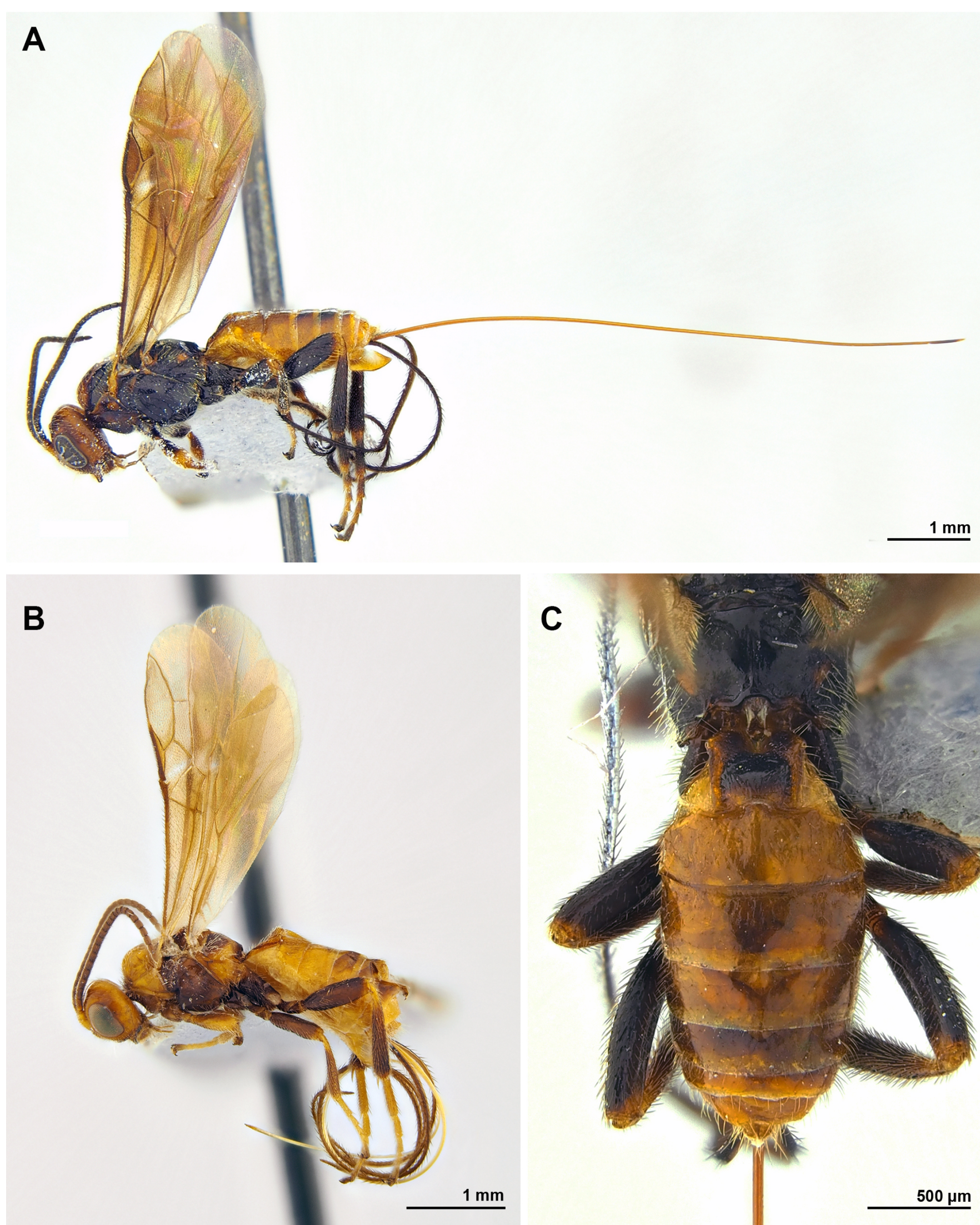

Figure 2. Bracon talyshicus Tobias, 1976; A. Female in lateral view (darker specimen); B. Female in lateral view (lighter specimen); C. Propodeum and metasoma in dorsal view. 


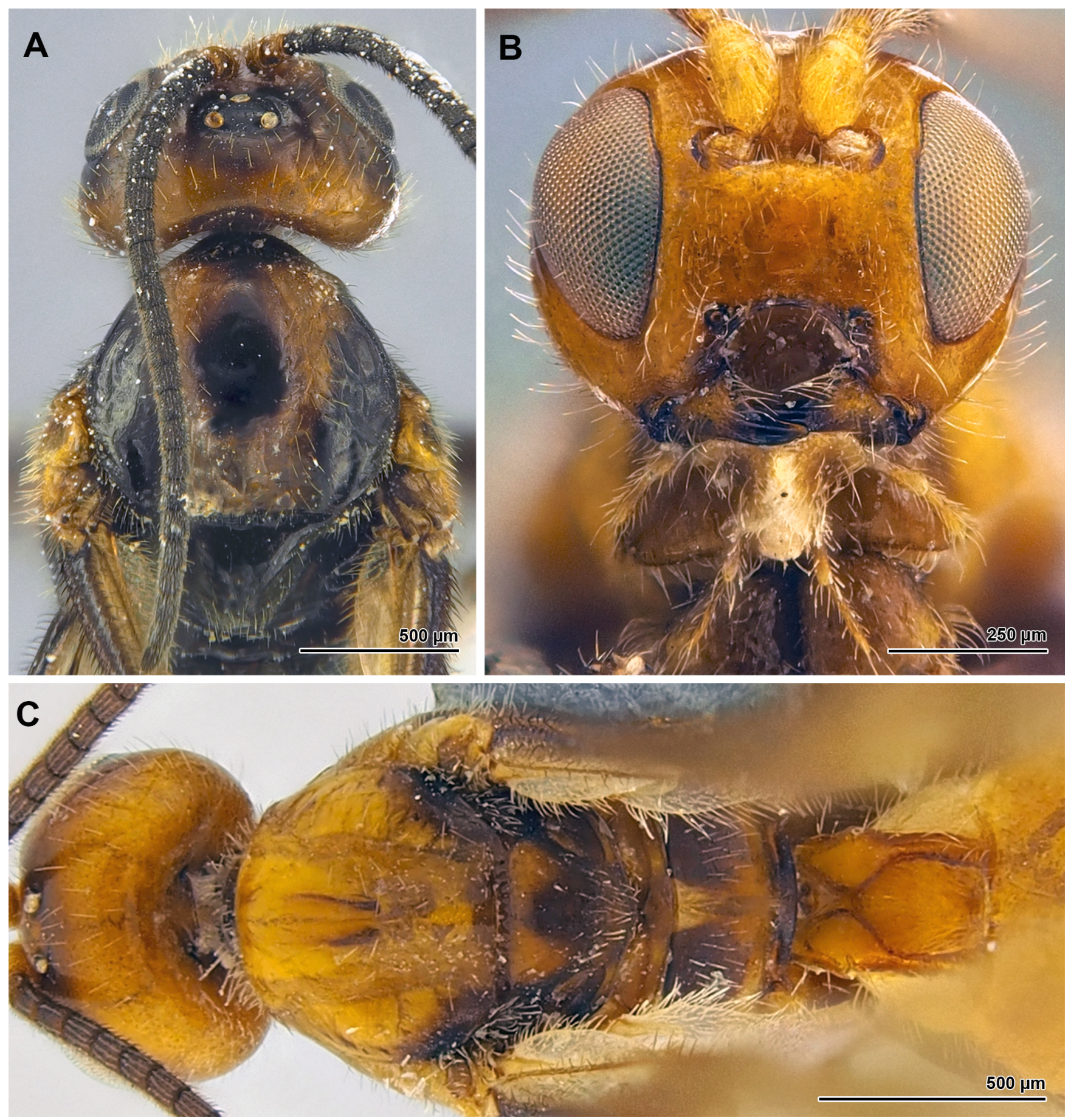

Figure 3. Bracon talyshicus Tobias, 1976; A. Head and mesosoma in dorsal view; B. Head in frontal view; C. Head, mesosoma and base of metasoma in dorsal view.

\section{DISCUSSION}

In the present study, 10 species of Braconinae were obtained from Asteraceae infested by nonfrugivorous Tephritidae. All host-parasitoid-plant associations are new (Table 1). There were not any host and host plant records for these species in the earlier literature (Yu et al., 2016). Redfern et al. (1992) obtained Bracon sp. on Terellia serratulae (L.) on Cirisium vulgare (Savi) in the UK. An unknown Bracon species was reared on Urophora solstitialis (L.) on Carduus nutans L. in Europe (Edwards et al., 1996), while we did not reared any braconids on Carduus spp. in our studied area. Urophora spp. was obtained with three braconids species (see Table 1). 
Table 1. Reared Braconidae on flower-heads of Asteraceae and associated Tephritidae in Iran (* new record).

\begin{tabular}{|c|c|c|c|}
\hline Bracon species & Host plants & Associated Tephritidae & References \\
\hline B. bipartitus Wesmeal, 1838 & $\begin{array}{l}\text { Lactuca serriola L. } \\
\text { Echinops sp. }\end{array}$ & $\begin{array}{l}\text { Hypenidium robrowskii (Becker, 1908) } \\
\text { Tephritomiya lauta (Loew, 1969) }\end{array}$ & Present study \\
\hline B. brevicornis (Wesmael, 1838) & Carthamus tinctorius L. & $\begin{array}{l}\text { Acanthiophilus helianthi (Rossi, 1794) } \\
\text { Chaetorellia carthami Stackelberg, } 1929 \\
\text { Terellia luteola Wiedemann, } 1830 \\
\text { Urophora mauritanica (Macquart, 1851) }\end{array}$ & $\begin{array}{l}\text { Lotfalizadeh \& } \\
\text { Gharali (2014) }\end{array}$ \\
\hline B. leptus Marshall, 1897 & Cousinia sp. & not found & Present study \\
\hline \multirow[t]{4}{*}{ B. luteator Spinola, 1808} & $\begin{array}{l}\text { Cirsium spectabile DC. } \\
\text { Echinops sp. } \\
\text { Cirsium ciliatum (Murray) } \\
\text { Onopordum sp. } \\
\text { Centaurea iberica Trevir \& Spreng } \\
\text { Onopordum acanthium L. } \\
\text { Cirsium vulgare (Savi) Ten. } \\
\text { Arctium lappa L. }\end{array}$ & $\begin{array}{l}\text { not found } \\
\text { not found } \\
\text { Xyphosia mililaria (Schrank, 1781) } \\
\text { Tephritis postica (Loew, 1844) } \\
\text { Urophora sp. } \\
\text { Chaetorellia carthami Stackelberg, } 1929 \\
\text { Chaetorellia conjuncta }(\text { Becker, 1913) } \\
\text { Tephritis postica (Loew, 1844) } \\
\text { Terellia serratula (L., 1758) } \\
\text { Tephritomiya lauta (Loew, 1969) }\end{array}$ & Present study \\
\hline & Carthamus tinctorius L. & $\begin{array}{l}\text { Acanthiophilus helianthi (Rossi, 1794) } \\
\text { Chaetorellia carthami, Stackelberg, } 1929 \\
\text { Terellia luteola Wiedemann, } 1830 \\
\text { Urophora mauritanica (Macquart, 1851) }\end{array}$ & $\begin{array}{l}\text { Lotfalizadeh \& } \\
\text { Gharali (2014) }\end{array}$ \\
\hline & & Some Tephritidae & Falcoj et al. (1993) \\
\hline & $\begin{array}{l}\text { Centaurea spp. } \\
\text { Oirsium spp. } \\
\text { Carduus spp. }\end{array}$ & Urophora solstitialis (L., 1758) & $\begin{array}{l}\text { Beyarsalan et al. } \\
\text { (2005); Tobias } \\
(1986)\end{array}$ \\
\hline B. mariae Dalla Torrer, 1898 & $\begin{array}{l}\text { Echinops sp. } \\
\text { Cirsium ciliatum (Murray) } \\
\text { Onopordum sp. } \\
\text { Centaurea iberica Trevir \& Spreng }\end{array}$ & $\begin{array}{l}\text { not found } \\
\text { not found } \\
\text { Tephritis postica (Loew, 1844) } \\
\text { Urophora sp. } \\
\text { Chaetorellia carthami Stackelberg, } 1929 \\
\text { Chaetorellia conjuncta (Becker, 1913) }\end{array}$ & Present study \\
\hline B. pectoralis Wesmael, 1838 & Carlina vulgaris L. & not found & Present study \\
\hline \multirow[t]{2}{*}{ B. subrugosus Szepligeti, 1901} & $\begin{array}{l}\text { Cirsium echinus (M.Bieb.) Hand.- } \\
\text { Mazz }\end{array}$ & Tephritis cometa (Loew, 1840) & Present study \\
\hline & $\begin{array}{l}\text { Angelica sylvestris } \mathrm{L} . \\
\text { Daucus carota } \mathrm{L} . \\
\text { Peucedanum arenarium } \mathrm{L} . \\
\text { Pimpinella saxifraga } \mathrm{L} .\end{array}$ & $\begin{array}{l}\text { Chaetostomella cylindrica (Robineau- } \\
\text { Desvoidy, 1830) } \\
\text { Noeeta pupillata (Fallén, 1814) } \\
\text { Tephritis leontodontis (De Geer, 1776) } \\
\text { Terellia serratulae (L., 1758) } \\
\text { Urophora solstitialis (L., 1785) } \\
\text { Urophora cardui (L., 1758) } \\
\text { Urophora quadrifasciata (Meigen, 1826) }\end{array}$ & Beyarslan (2014) \\
\hline B. talyshicus Tobias, $1976^{*}$ & $\begin{array}{l}\text { Cirsium echinus (M.Bieb.) Hand.- } \\
\text { Mazz } \\
\text { Echinops sp. }\end{array}$ & $\begin{array}{l}\text { not found } \\
\text { Tephritomiya lauta (Loew, 1969) }\end{array}$ & Present study \\
\hline B. trucidator Marshall, 1888 & $\begin{array}{l}\text { Cirsium arvensis (L.) Scop. } \\
\text { Cirsium sp. } \\
\text { Onopordum acanthium } \mathrm{L} . \\
\text { Arctium lappa } \mathrm{L} . \\
\text { Onopordum sp. }\end{array}$ & $\begin{array}{l}\text { Chaetorellia sp. } \\
\text { Terellia nigripalpis Hendel, } 1927 \\
\text { Urophora sp. } \\
\text { Tephritis bardanae (Schrank, 1830) } \\
\text { Urophora terebrans (Loew, 1850) }\end{array}$ & Present study \\
\hline
\end{tabular}


Table 1. Continued.

\begin{tabular}{llll}
\hline B. trucidator Marshall, 1888 & Carduus nutans L. & $\begin{array}{l}\text { Myopites inulaedyssenteriae Blot, 1827 } \\
\text { Urophora solstitialis (L., 1758) }\end{array}$ & Beyarslan (2014) \\
\hline B. tschitscherini Kokujev, 1904 & Echinops sp. & Tephritomiya lauta (Loew, 1969) & Present study \\
\hline B. urinator (Fabricius, 1798) & Cirsium spectabile DC. & not found & Present study \\
& Cirsium congestum Fisch. \& & Tephritomiya lauta (Loew, 1969) & \\
& C.A.Mey. & Tephritis pulchar (Loew, 1844) & Zikic et al. (2012) \\
\cline { 2 - 4 } & Cirsium sp. & & \\
& Echinop sp. & & \\
& Prangos sp. & & \\
& Carduus sp. & & \\
& Scorzonera sp. & & \\
& Centaurea sp. & & \\
& Tragopogon sp. & &
\end{tabular}

The previously recorded data were mainly concentered on the economically important tephritid species that were reared on safflower fruit flies, Carthamus tinctorius L., as a key pest of safflower. Lotfalizadeh \& Gharali (2014) reported Bracon hebetor Say, B. brevicornis (Wesmael) and B. luteator Spinola and Basheer et al. (2014) reported B. luteator and B. intercessor Nees in Iran and Syria, respectively. Bracon luteator was a most abundant species in safflower fields in Iran (Lotfalizadeh \& Gharali, 2014).

Some species of two other braconids of Opiinae and Alysiinae have also been reported with nonfrugivorous Tephritidae, infesting Asteraceae (Wharton \& Yoder, 2014), were not found in the present study. So far 115 species of the subfamily Braconinae from Iran (Farahani et al. 2016), of which, 96 species belonging to six subgenera, included in the genus Bracon (Zargar et al., 2020). A new record of B. talyshicus increases it to 97 species. Within the reported species in this research, B. talyshicus and $B$. bipartitus, were reared for the first time on Echinops sp. and Lactuca serriola (Astraceae) that infested with Tephritomyia lauta and Hypenidium robrowskii (Dipt.: Tephritidae), respectively. Within reared Bracon species on Asteraceae, B. luteator and B. urinator have a wide range of associated host plants, reared from nine species (Table 1). Bracon luteator and B. trucidator were found in association with numerous Tephritids (11 and 7 species, respectively - see Table 1). Bracon luteator has already been reported on non-frugivorous Tephritidae flies by Lotfalizadeh \& Gharali (2014) that confirm this plantphytophagous-parasitoid relationship. However, most of plant-phytophagous-parasitoid associations are new. For confirmation of host-parasitoid association, further studies with separately rearing of infested larvae or pupa of non-frugivorous Tephritidae can be helpful.

\section{AUTHOR'S CONTRIBUTION}

The authors confirm contribution to the paper as follows: A.P.: collecting, rearing and writing of manuscript. H.L.: designing research plane, writing of manuscript. N.K.: identification of host plants. B.G.: identification of fruit flies. A.A.: identification of some Bracon species.

\section{FUNDING}

This research received no specific grant.

\section{AVAILABILITY OF DATA AND MATERIAL}

Not applicable.

\section{ETHICS APPROVAL AND CONSENT TO PARTICIPATE}

Not applicable. 


\section{CONSENT FOR PUBLICATION}

Not applicable.

\section{CONFLICT OF INTERESTS}

The authors declare that there is no conflict of interest regarding the publication of this paper.

\section{ACKNOWLEDGMENTS}

We are grateful to Dr. J. Yarahmadi (East-Azarbaijan Agricultural and Natural Resources Research Center, Agricultural Research, AREEO, Tabriz, Iran) and Dr. M. Asadi (Department of Plant Protection, Faculty of Agriculture, Urmia University, Urmia, Iran) their valuable contribution for preparation of distribution map of studied species, Dr. K. Samartsev (Zoological Institute of the Russian Academy of Sciences, Saint Petersburg, Russia) for confirmation and correction of identifications.

\section{REFERENCES}

Abdoli, P., Talebi, A.A. \& Farahani, S. (2019a) Dolichogenidea fernandeztrianai sp. nov. (Hymenoptera: Braconidae, Microgastrinae) from Iran. Journal of Agricultural Science and Technology, 21 (3), 647-658.

Abdoli, P., Talebi, A.A., Farahani, S. \& Fernandez-Triana, J. (2019b) Three new species of the genus Choeras Mason, 1981 (Hymenoptera: Braconidae, Microgastrinae) from Iran. Zootaxa, 4545 (1), 77-92. https:/ / doi.org/10.11646/zootaxa.4545.1.4

Abdoli, P., Talebi, A.A., Fernandez-Triana, J. \& Farahani, S. (2021a) Taxonomic study of the genus Microplitis Förster, 1862 (Hymenoptera, Braconidae, Microgastrinae) from Iran. European Journal of Taxonomy, 744 (1), 83-118. https://doi.org/10.5852/ejt.2021.744.1305

Abdoli, P., Talebi, A.A., Fernandez-Triana, J. \& Farahani, S. (2021b) Description of a new species of the genus Protapanteles Ashmead, 1898 (Hymenoptera: Braconidae: Microgastrinae) from Iran. Annales Zoologici, 71 (2), 289-295. https://doi.org/10.3161/00034541ANZ2021.71.2.007

Aguiar, A.P., Deans, A.R., Engel M.S., Forshage, M., Huber, J.T., Jennings, J.T., Johnson, N.F., Lelei, A.S., Longino, J.T., Lohrmann, V., Miko, I., Ohl, M., Rasmussen, C., Taeger, A. \& Yu, D.S.K. (2013) Order Hymenoptera. Zootaxa, 3703, 51-62. https://doi.org/10.11646/zootaxa.3703.1.12

Ameri, A., Talebi, A.A., Beyarslan, A., Kamali, K. \& Rakhshani, E. (2014) Study of the genus Bracon Fabricius, 1804 (Hymenoptera: Braconidae) of Southern Iran with description of a new species. Zootaxa, 3754 (4), $353-380$. https://doi.org/10.11646/zootaxa.3754.4.2

Ameri, A., Talebi, A.A., Rakhshani, E., Beyarslan, A. \& Kamali, K. (2015) Additional evidence and new records of the genus Bracon Fabricius, 1804 (Hymenoptera: Braconidae) in southern Iran. Turkish Journal of Zoology, 39, 11101120. https://doi.org/10.3906/zoo-1404-62.

Ameri, A., Ebrahimi, E. \& Talebi. A.A. (2020) Additions to the fauna of Braconidae (Hym.: Ichneumonoidea) of Iran based on the specimens housed in Hayk Mirzayans Insect Museum with six new records for Iran. Journal of Insect Biodiversity and Systematics, 6 (4), 353-364.

Basheer, A., Asslan, L. \& Abdalrazaq, F. (2014) Survey of the parasitoids of the tephritid fruit flies of the safflower Carthamus tinctorius (Asteracea) in Damascus, Syria. Egyptian Journal of Biological Pest Control, 24 (1), $169-172$.

Beyarslan, A. \& Fischer, M. (1990) Bestimmungsschl“ussel zur Identifikation der palaarktischen Bracon-Arten des Subgenus Glabrobracon Tobias (Hym.: Braconidae: Braconinae). Annalen des Naturhistorischen Museums in Wien, 91B, 137-145.

Beyarslan, A., Çetin-Erdoğan, Ö. \& Aydogdu, M. (2005) A Survey of Braconinae (Hymenoptera, Braconidae) of Turkish Western Black Sea Region. Linzer biologische Beitrage, 37 (1), 195-213.

Beyarslan, A. (2014) Checklist of Braconinae species of Turkey (Hymenoptera: Braconidae). Zootaxa, 3790 (2), $201-$ 242. https:/ / doi.org/10.11646/zootaxa.3790.2.1

Čapek, M. \& Zwolfer, H. (1990) Braconids (Hymenoptera, Braconidae) associated with insects inhabiting thistles (Asteraceae, Cynaroideae). Acta Entomological Bohemoslovaca, 87 (4), 262-277. 
Dolati, S., Talebi, A.A., Peris-Felipo, F. J., Farahani, S. \& Khayrandish, M. (2021) New data on the subfamily Opiinae (Hymenoptera: Braconidae) from Iran. Zootaxa, 4903 (3), 331-352. https:/ / doi.org/10.11646/ zootaxa.4903.3.2

Edwards, P.B., Adair, R.J. \& Holtkamp, R.H. (1996) Parasitoids- can predictions be made about likely parasitism of tephritids introduced into Australia for biological control of Asteraceae?. In: Maroran, V.C. \& Hoffmann, J.H. (eds) Proceedings of the IX International Symposium on Biological Control of Weeds, 1996, 19-26, Stellenbosch, South Africa, University of Cape Town, pp. 153-164.

Falco, V., Monero, J. \& Jimenez, R. (1993) Datos sobre Ciclostominos ibéricos. I. Braconinae (Hymenoptera, Braconidae). Boletin de la Asociacion Espanola de Entomologia, 17 (1), 71-90.

Farahani, S., Talebi, A.A. \& Rakhshani, E. (2016) Iranian Braconidae (Insecta: Hymenoptera: Ichneumonoidea): diversity, distribution and host association. Journal of Insect Biodiversity and Systematics, 2 (1), 1-92.

Gates, M., Mena-Correa, J., Sivinski, J., Ramírez-Romero, R., Córdova-García, G. \& Aluja, M. (2008) Description of the immature stages of Eurytoma sivinskii Gates and Grissell (Hymenoptera: Eurytomidae), an ectoparasitoid of Anastrepha (Diptera: Tephritidae) pupae. Entomological News, 119, 354-360. https://doi.org/10.3157/0013-872X-119.4.354

Ghahari, H. \& Fischer, M. (2011) A study on the Braconidae (Hymenoptera: Ichneumonoidea) from some regions of northern Iran. Entomofauna, 32 (8), 181-196.

Korneyev, V.A. \& White, I.M. (1999) Tephritids of the genus Urophora R.-D. (Diptera: Tephritidae) of East Palaearctic. III. Key to Palaearctic species. Entomological Review, 79 (3), 464-482.

Lotfalizadeh, H. \& Gharali, B. (2014) Hymenopterous parasitoids of Carthamus tinctorius L. seed pests in Iran. Applid Entomology and Phytopathology, 42, 47-62.

Mohammadzade-Namin \& Korneyev, V. (2018) An annotated checklist of fruit flies (Dip.: Tephritidae) of Iran. Zootaxa, (3), 377-405. https:// doi.org/10.11646/zootaxa.4369.3.5

Norrbom, A.L. (2010) Tephritidae (fruit flies, moscas de frutas). In: Brown, B.V., Borkent, A., Cumming, J.M., Wood, D.M., Woodley, N.E. \& Zumbado, M.A. (eds) Manual of Central American Diptera, volume 2. NRC Research Press, Ottawa, pp. 909-954.

Ovruski, S.M., Schliserman, P. \& Aluja, M. (2004) Indigenous parasitoids (Hymenoptera) attacking Anastrepha fraterculus and Ceratitis capitata (Diptera: Tephritidae) in native andexotic host plants in Northwestern Argentina. Biological Control, 29, 43-57. https:/ / doi.org/10.1016/S1049-9644(03)00127-0

Papp, J. (1966) A synopsis of the Bracon F. species of the Carpathian Basin (Hymenoptera, Braeonidae) I. Subgenus Glabrobracon Fahr. Annalks Historico-Naturales Musei Nationalis Hungarici, 58, 373-394.

Papp, J. (1968) A synopsis of the Bracon Fabr. species of the Carpathian Basin, Central Europe (Hymenoptera, Braconidae) II. Subgenus Bracon Fabr. Annales Historico-Naturales Musei Nationalis Hungarici, 60, $191-211$.

Papp, J. (1969a) A revision of Thomson's species of Bracon Fabr. (Hymenoptera, Braconidae). Opuscula Entomologica, 34, 177-205.

Papp, J. (1969b) A synopsis of the Bracon Fabr. species of the Carpathian Basin (Hymenoptera, Braconidae), III. Subgenus Lucobracon. Annales Historico-Naturales Musei Nationalis Hungarici, 61, 317-335.

Pourhaji, A., Lotfalizadeh, H., Farshbaf-Pourabad, R., Gharali, B. \& Mohammadi-Khoramabadi, A. (2016) Ichneumonid parasitoids (Hymenoptera: Ichneumonidae) of the non frugivorous Tephritidae flies (Diptera: Tephritidae) in the northwest of Iran. Journal of Insect Biodiversity and Systematics, 2 (2), 193-202.

Pourhaji, A., Lotfalizadeh, H., Farshbaf-Pourabad, R. \& Gharali, B. (2020) Parasitic wasps (Hymenoptera: Chalcidoidea) associated with flower-heads of Asteraceae in Iran. Biologia, 75, 547-565. https://doi.org/10.2478/s11756-019-00319-7

Rahmani, Z., Rakhshani, E., Konstantin G. Samartsev, K.S. \& Mokhtari, A. (2017) A survey of the genera Bracon Fabricius, 1804 and Habrobracon Ashmead, 1895 (Hymenoptera, Braconidae, Braconinae) in Iran. Turkish Journal of Zoolgy, 41, 821-840. https:/ / doi.org/10.3906/zoo-1611-68

Redfern, M., Jones, T.H. \& Hassell, M.P. (1992) Heterogeneity and density dependence in a filed study of a tephritidparasitoid interaction. Ecological Entomology, 17, 255-262. https://doi.org/10.1111/j.1365-2311.1992.tb01056.x

Sharkey, M.J., Janzen, D.H., Hallwachs, W., Chapman, E.G., Smith, M.A., Dapkey, T., Brown, A., Ratnasingham, S., Naik, S., Manjunath, R., Perez, K., Milton, M., Hebert, P., Shaw, S.R., Kittel, R.N., Solis, M.A., Metz, M.A., 
Goldstein, P.Z., Brown, J.W., Quicke, D.L.J., van Achterberg, C., Brown, B.V. \& Burns, J.M. (2021) Minimalist revision and description of 403 new species in 11 subfamilies of Costa Rican braconid parasitoid wasps, including host records for 219 species. ZooKeys, 1013, 1-665. https:/ / doi.org/10.3897/zookeys.1013.55600

Shaw, S.R. \& Edgerly, J. (1986) A new braconid genus parasitizing web spinners (Embiidina) in Trinidad. Psyche, 92, 505-511. https://doi.org/10.1155/1985/54285

Shaw, M.R. \& Huddleston, T. (1991) Classification and biology of braconid wasps (Hymenoptera: Braconidae). Handbooks for the Identification of British Insects, 7 (11), 1-126.

Starý, P. (1970) Biology of Aphid Parasites (Hym.: Aphidiidae) with respect to Integrated Control. Dr. W. Junk, B.V., The Hague, 643 pp.

Sivinski, J., Piñero, J. \& Aluja, M. (2000) The distributions of parasitoids (Hymenoptera) of Anastrepha non frugivorous Tephritidae flies (Diptera: Tephritidae) along an altitudinal gradient in Veracruz, Mexico. Biological Control, 18, 258-269. https:/ / doi.org/10.1006/bcon.2000.0836

Tobias, V.I. (1986) Gnaptodontinae, Braconinae, Telengainae. In: Medvedev, G.S. (ed) Opredelitel Nasekomych Evrospeiskoi Tsasti SSSR 3, Peredpontdatokrylye 4. Opred. Faune SSSR, pp. 85-149.

Uchôa, M.A. \& Niccio, J.N. (2010) New records of Neotropical non frugivorous Tephritidae flies (Tephritidae), lance flies (Lonchaeidae) (Diptera: Tephritoidea), and their host plants in the South Pantanal and adjacent areas, Brazil. Annals of the Entomological Society of America, 103 (5), 723-733. https:/ / doi.org/10.1603/ AN09179

van Achterberg, C. (1993) Illustrated key to the subfamilies of the Braconidae (Hymenoptera: Ichneumonoidea). Zoologische Verhandelingen Leiden, 283, 1-189.

van Achterberg, C. (2014) Notes on the checklist of Braconidae (Hymenoptera) from Switzerland. Mitteilungen der Schweizerischen Entomologischen Gesellschaft, 87, 191-213.

Varis, A.L. \& van Achterberg, C. (2001) Peristenus varisae spec.nov. (Hymenoptera: Braconidae) parasitizing the European tarnished plant bug, Lygus rugulipennis Poppius (Heteroptera: Miridae). Zoologische Verhandelingen (Leiden), 75, 371-38.

Wharton, R.A. (1993) Bionomics of the Braconidae. Annual Review of Entomology, 38, 21-143. https:/ / doi.org/10.1146/annurev.en.38.010193.001005

Wharton, R.A., Ovruski, S.M. \& Gilstrap, F.E. (1998) Neotropical Eucoilidae (Cynipoidea) associated with non frugivorous Tephritidae infesting Tephritidae, with new records from Argentina, Bolivia and Costa Rica. Journal Hymenoptera Research, 7, 102-115.

Wharton, R.A. \& Yoder, M.J. (2014) Parasitoids of non frugivorous Tephritidae-infesting Tephritidae. Available from: $h$ ttp://paroffit.org/ (Accessed 02th April 2017).

Yu, D.S., van Achterberg, C. \& Horstmann, K. (2016) Taxapad 2016 - World Ichneumonoidea 2015. Taxonomy, Biology, Morphology and Distribution. Ontario: Nepean, Canada. On USB Flash Drive.

Zargar, M., Talebi, A.A., Hajiqanbar, H. \& Papp, J. (2015) A study on the genus Bracon Fabricius (Hymenoptera: Braconidae) in north central Iran with four new records for Iranian fauna. Entomofauna, 36 (32), $425-440$.

Zargar, M., Samartsev, K., Talebi, A.A. \& Farahani, S. (2020) Study of the genus Bracon Fabricius, 1804 (Hymenoptera: Braconidae) from Iran: new subspecies, new records and an updated checklist. Zootaxa, 4758 (2), 201-230. https://doi.org/10.11646/zootaxa.4758.2.1

Zikic, V., Stankovic, S.S. \& Ilic, M. (2012) Checklist of the genus Bracon (Hymenoptera: Braconidae) in Serbia. Biologica Nyssana, 3 (1), 21-29. 
زنبورهاى جنس Hymenoptera: Braconidae) Bracon Fabricius) مرتبط با طبق تياهان خانواده در ايران

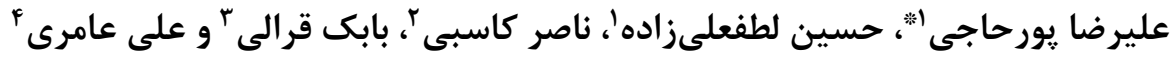 \\ ا گروه كَياهيزشكى، مركز تحقيقات كشاورزى و منابع طبيعى استان آذربايجان شرقى، تحقيقات كشاورزى، تبريز، ايران.

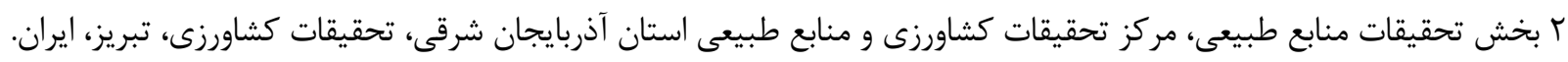

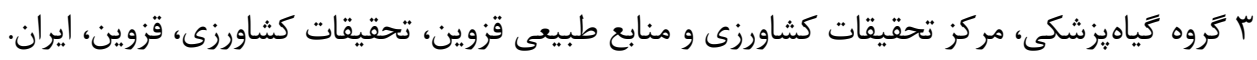

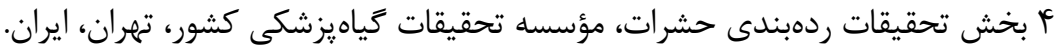

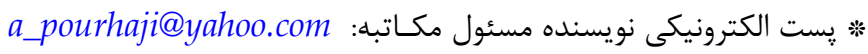

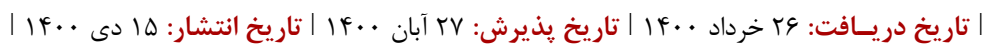 \\ جكيـده: اين تحقيق به منظورجمعآورى و شناسايى زنبورهاى Braconidae مرتبط با

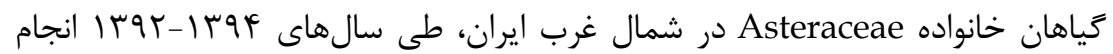

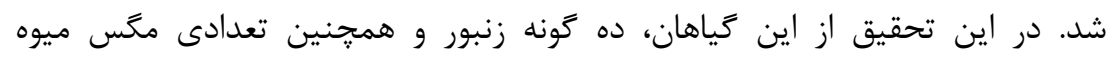

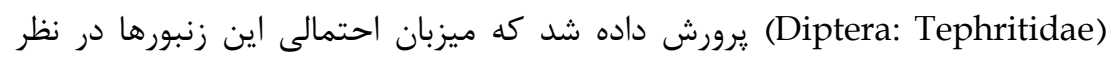

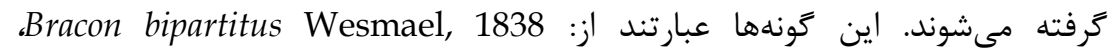 \\ B. mariae Dalla Torre, ،B. luteator Spinola, 1808 ،B. leptus Marshall, 1897 \\ ،B. subrugosus Szépligeti, 1901 ،B. pectoralis Wesmael, 1838 ، 1898 \\ B. tschitscherini ‘B. trucidator Marshall, 1888 ،B. talyshicus Tobias, 1976 \\ B. urinator (Fabricius, 1798) و كه از بين آنها كونه \\ B. talyshicus

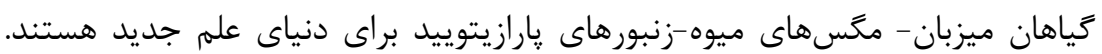

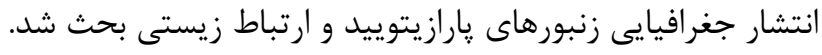 \\ وازَّــان كليدى: ميزبان، انتشار، يارازيتوييد، Braconidae، ايران
}

